\title{
Concerted motion of protons in hydrogen bonds of DNA-type molecules.
}

\author{
V.L.Golo ${ }^{1,3}$, E. I. Kats ${ }^{2,4}$, M.Peyrard ${ }^{3}$ \\ 1 Department of Mechanics and Mathematics \\ Moscow University, 119899 Moscow, Russia ; \\ ${ }^{2}$ Laue-Langevin Institute, F-38042, Grenoble, France ; \\ ${ }^{3}$ Lab. Phys. ENS Lyon, 69364, Lyon, Cedex 07, France; \\ ${ }^{4}$ L. D. Landau Institute for Theoretical Physics, RAS \\ 117940, Kosygina 2, Moscow, Russia.
}

(October 26, 2018)

\begin{abstract}
We study the dynamical behaviour of the proton transfer in the hydrogen bonds in the base-pairs of the double helices of the DNA type. Under the assumption that the elastic and the tunnelling degrees of freedom may be coupled, we derive a non-linear and non-local Schrodinger equation (SNLNL) that describes the concerted motion of the proton tunnelling. Rough estimates of the solutions to the SNLNL show an intimate interplay between the concerted tunnelling of protons and the symmetry of double helix.

PACS numbers: $87.15-\mathrm{v}, 87.14 . \mathrm{Gg}$
\end{abstract}

Typeset using REVTEX 
I.

The recent direct observation of coherent proton tunnelling in macromolecules [1] has focused the attention on systems allowing for the nontrivial dynamics of protons contained in hydrogen bonds. Perhaps, the most significant system of the kind is the DNA molecule. The problem of the proton transfer inside the hydrogen bonds of the double helix representing a molecule of DNA, has a long and rich history. The importance of the phenomenon was noticed soon after the DNA double helix had been discovered (see [2]). In fact from the chemical point of view the proton transfer is a so-called tautomerization reaction, that is a kind of transition that preserves the constituent atoms of a compound but at the same time changes their mutual positions. It has been believed that the tautomerism transition could provide a mechanism for genetic mutations [2].

Later Crick [3] suggested that the mutations could be due to conformational changes within the double helix, the so-called "wobbling" [2]. By now there has been no definite conclusion as to whether the wobbling or the tautomerism are responsible for the occurrence of mutations. The recent work on the i-motif of DNA, in which the tautomerism, rare in the usual DNA, is the rule, and not an exception [4] has given a new impetus to the problem of proton transfer.

The phenomenon of tautomerism in the base pairs of DNA has been studied extensively, both experimentally and theoretically [2], [5] , [6], but for individual single molecules of purines and pyrimidines. Here it should be noted that even though one has got a considerable body of information about the chemical reaction corresponding to the tautomer transition, such as its constant of reaction, and even managed to find an estimate for the concentration of tautomeric forms, $10^{-4}-10^{-5} \mathrm{~mol} / \mathrm{l}$, for the B-type of DNA, there is a little knowledge as far as the dynamics of the proton transfer accompanying it in DNA is concerned. In fact, the estimates for the transition frequency widely diverge, and generally it is believed to be within $10^{6}-10^{11} s^{-1}$, [4].

In our opinion the unsatisfactory state of the art as regards to the proton transfer, and the tautomeric dynamics, in the DNA, is to a certain extent due to the absence of theoretical 
models of the phenomenon. In the present paper we would like to draw attention to the fact that if the tautomeric transitions are coupled with a change in elastic properties of the DNA molecule, one could expect a concerted tunnelling of the protons in the hydrogen bonds. Taking into account the extremely sophisticated nature of the system, i.e. the DNA molecule, we aim at studying it in a qualitative framework, which takes into account, the main features of the molecule, i.e. the presence of the two strands, the helical structure, or the winding symmetry, and the dynamics of protons considered within the approximation of two-level systems.

II.

Aiming at a simple model, we shall describe the states of the hydrogen bonds as those of a one-dimensional Bose oscillator, the two-level requirement being accommodated by considering only its ground state and the lowest excited one. In this sense the tautomeric reaction of the proton transfer is described by the excited state of an oscillator. Thus, we shall consider the protons inside the hydrogen bonds of a molecule of DNA as a quantum system described by the Bose operators $b_{n}^{+}, b_{n}$ subject to the usual commutation relation

$$
\left[b_{n}^{+}, b_{m}\right]=\delta_{n m}
$$

in which $n, m$ are the indices of the corresponding sites of hydrogen bonds. We shall suppose that the system of the protons is in a weakly excited state that can be cast in the form of wave function

$$
\left|D(t)>=\sum_{n} A_{n}(t) b_{n}^{+}\right| 0>
$$

in which $A_{n}(t)$ are complex amplitudes subject to the relation

$$
\sum_{n}\left|A_{n}\right|^{2}=1
$$

The Hamiltonian of the total system, that is the protons and the elastic part corresponding to the sugar-phosphates, reads

$$
H=H_{H}+H_{Y}+H_{I}
$$


where $H_{H}$ is the proton Hamiltonian

$$
H_{H}=\sum_{n} E_{0} b_{n}^{+} b_{n}-\kappa \sum_{n}\left(b_{n+1}^{+} b_{n}+b_{n}^{+} b_{n+1}\right)
$$

where $E_{0}$ is the level splitting between two states of the proton in a hydrogen bond, and $\kappa$ is the tunnelling probability. $H_{Y}$ is that of the elastic part, and $H_{I}$ is the interaction between them. We shall consider the elastic part of the system as the classical one, and even neglect its kinetic energy. The reason is that the characteristic times for the elastic vibrations of the DNA molecule are usually estimated as being in the region of $10^{-11}-10^{-13} \mathrm{~s}$, whereas the proton tunnelling is alleged to be within $10^{-6}-10^{-11} \mathrm{~s}$ (more exact figures are unavailable; according to [5] the value is $10^{-6} \mathrm{~s}$ ). Thus, one may suggest that the elastic motion is following the proton tunnelling without inertia so that we may take into account the lattice deformation only through the potential energy $H_{Y}$, neglecting the kinetic one. In writing $H_{Y}$ we shall follow the method worked out in papers [7], 8], aiming at a simplified description of the dynamics of the double helix which is considered as a one-dimensional lattice of vectors $\mathbf{y}_{n}$ that describe the mutual positions of the two strands at sites corresponding to the base pair of index $n$. The helix structure is described with the help of the covariant derivative for the description of deformations resulting from the change in the positions of the strands, so that the potential $H_{Y}$ reads

$$
H_{Y}=\sum_{n}\left[\frac{K}{2}\left(\nabla \mathbf{y}_{n}\right)^{2}+\frac{\epsilon}{2} \mathbf{y}_{\mathbf{n}}^{2}\right]
$$

Here the harmonic term $(1 / 2) \epsilon y_{n}^{2}$ describes the binding of the two strands, and $\nabla \mathbf{y}_{n}$ is the covariant derivative in discrete form, linearized with respect to rotations that relates the two adjacent sites, $n$ and $n+1$.

$$
\nabla \mathbf{y}_{n} \equiv \frac{1}{a}\left(\mathbf{y}_{n+1}-\mathbf{y}_{n}+\hat{\Omega} \mathbf{y}_{n}\right)
$$

and

$$
\Omega_{i j}=-\epsilon_{i j k} \Omega_{k}
$$


where $\Omega_{k} \equiv(0,0, \Omega), K, \epsilon$ are elastic constants, and $a$ is the spacing of the lattice of the base pairs. We are assuming that the molecule is parallel to the $z$ axis. Having chosen the dynamical variables $b_{n}^{+}, b_{n}, \mathbf{y}_{n}$ for the protons and the elastic excitations, we have a limited number of option for the interaction energy. The simplest one should couple the proton excited states given by $b_{n}^{+} b_{n}$, and the elastic deformations, which are a function of $\mathbf{y}_{n}$, in the simplest situation we adopt, the linear one. We assume that $\mathbf{y}_{n}$ enters in the form of its covariant derivative, for this way one might accommodate the interaction of the proton tunnelling with the stack of the $\pi$-electrons of the bases.

The choice of the interaction Hamiltonian is delicate but the knowledge of the structure and binding in DNA gives some indications. The stacking interaction between adjacent base pairs is strongly affected by the overlap of the $\pi$ electrons of the bases. But the proton transfer is accompanied by a redistribution of the electrons on the bases so that the stacking interaction is changed and therefore we expect that the proton tunneling will affect the coupling between adjacent bases. It means that the derivative $\nabla \mathbf{y}_{n}$ rather than $\mathbf{y}_{n}$ itself should enter in the interaction Hamiltonian. We have chosen the simplest form coupling the commponent of the gradient which is along the direction $\mathbf{h}_{n}$ of the hydrogen bonds connecting the bases within pair $n$ and the state of the protons in this pair given by $b_{n}^{+} b_{n}$, i.e.

$$
H_{I}=-\lambda \sum_{n}\left(\nabla \mathbf{y}_{n} \cdot \mathbf{h}_{n}\right) b_{n}^{+} b_{n},
$$

in which the vectors $\mathbf{h}_{n}$ are subject to the helical symmetry of the molecule. They can be written in the form

$$
\mathbf{h}_{n}=(\cos n \alpha ; \sin n \alpha ; 0),
$$

where the angle $\alpha$ is related to the helical pitch. Having chosen a primitive model of the DNA, and neglecting the subtle features like 2 or 3 hydrogen bonds for the A - T or G $\mathrm{C}$ base pairs, and confining ourselves to one-site one H-bond picture, we may set $\alpha=\Omega$ equalizing the pitch of the $\mathbf{h}_{n}$ and the twist of the double helix. 
It is important to notice that the sign of $\lambda$ will be crucial for the SNLNL equation that we shall derive. Our choice means that the stacking energy is reduced when protons are in the excited state.

We employ the method worked out by Davydov [9] to study the model described above. According to [9],

- we have to calculate the effective potential

$$
U_{\text {eff }}=<D(t)\left|H_{Y}+H_{I}\right| D(t)>
$$

which is a function of $\mathbf{y}_{n}$,

- find its minimum, $\mathbf{y}_{n}^{0}$,

- substitute $\mathbf{y}_{n}^{0}$ into $H$ given by Eq. (2), thus obtaining the effective Davydov Hamilto$\operatorname{nian} H_{D}$.

Finally, we have to write down the Schrodinger equation for the function $|D(t)\rangle$, given by Eq. (1), and the Hamiltonian $H_{D}$

$$
i \hbar \frac{\partial}{\partial t}\left|D(t)=H_{D}\right| D(t)>
$$

Both sides of the equation indicated above are linear forms in the operators $b_{n}^{+}$, and by equating the coefficients at corresponding $b_{n}^{+}$, we obtain the following equation for $A_{n}$

$$
\begin{gathered}
i \hbar \frac{\partial A_{n}}{\partial t}=E_{0} A_{n}-\kappa\left(A_{n+1}+A_{n-1}\right)-\frac{\lambda^{2}}{K}\left|A_{n}\right|^{2} A_{n}-\frac{\lambda^{2}}{K}\left[\sum_{m}\left|A_{m}\right|^{4}\right] A_{n}+ \\
\frac{\lambda^{2}}{2 K} \frac{\epsilon a^{2}}{K \Omega^{2}}\left\{\sum_{m^{\prime}, m^{\prime \prime}} \cos ^{\left|m^{\prime}-m^{\prime \prime}\right|} \phi \cos \left[\left(m^{\prime}-m^{\prime \prime}\right)(\phi-\Omega)\right]\left|A_{m^{\prime}}\right|^{2}\left|A_{m^{\prime \prime}}\right|^{2}\right\} A_{n}+ \\
\frac{\lambda^{2}}{2 K} \frac{\epsilon a^{2}}{K \Omega^{2}}\left\{\sum_{m} \cos ^{|m-n|} \phi \cos [(m-n)(\phi-\Omega)]\left|A_{m}\right|^{2}\right\} A_{n},
\end{gathered}
$$

in which the angle $\phi$ is determined by the equation $\tan \phi=\Omega$ and $\lambda / K$ and $\epsilon a^{2} /\left(K \Omega^{2}\right) \ll 1$ are small parameters. 
In assessing the importance of cos- terms in the equation given above, it is worthwhile to note that since we have assumed $\alpha=\Omega$ and there is the relation $\tan \phi=\Omega$, the typical term in the equation (11) contains a factor that reads asymptotically

$$
\exp \left(-\Omega^{2}|m-n|\right) \cos \left(|m-n| \Omega^{3}\right)
$$

We have assumed $\Omega$ being a small parameter, and therefore the oscillations due to $\Omega^{3}$ are negligible. Let us consider a possible simplification of the equation (11) obtained above. There is a chance that it may have a bearing on the dynamics of the proton transfer in DNA. To that end note that the angles $\phi$ and $\Omega$ are close to each other; indeed, for the $\mathrm{B}$ DNA the angle $\Omega$ corresponds to the pitch, i.e. 10 steps for $2 \pi$. For this reason, we set all the functions $\cos [(m-n)(\phi-\Omega)]$ equal to 1 in (11), and introduce

$$
A(z, t)=\exp \left(-i \frac{E_{0} t}{\hbar}\right) B(z, t)
$$

Then, in the continues notations, (i.e. for scales larger than $a$ ) we obtain the following non-linear and non-local Schrodinger equation $(z \equiv x / a)$.

$$
\begin{gathered}
i \frac{\partial B}{\partial t}=-\omega_{H} \frac{\partial^{2} B}{\partial z^{2}}-\frac{\lambda}{K} \omega_{T}\left|B^{2}\right| B-\frac{\lambda}{K} \omega_{T} B \int\left|B\left(z^{\prime}\right)\right|^{4} d z^{\prime}+ \\
\frac{\lambda}{K} \omega_{T} \frac{\epsilon a^{2}}{K \Omega^{2}}\left(\int d z^{\prime} \int d z^{\prime \prime} \exp \left(-\mu\left|z^{\prime}-z^{\prime \prime}\right|\right)\left|B\left(z^{\prime}\right)\right|^{2} \mid B\left(\left.z^{\prime \prime}\right|^{2}\right) B(z)+\right. \\
\frac{\lambda}{K} \omega_{T} \frac{\epsilon a^{2}}{K \Omega^{2}}\left(\int d z^{\prime} \exp \left(-\mu\left|z-z^{\prime}\right|\right) \mid B\left(\left.z^{\prime}\right|^{2}\right) B(z),\right.
\end{gathered}
$$

in which

$$
\mu=-\ln \cos \phi=\frac{1}{2} \ln \left(1+\Omega^{2}\right) \simeq \frac{\Omega^{2}}{2},
$$

and $\omega_{H} \equiv \kappa / \hbar, \omega_{T} \equiv \lambda / \hbar$. We may perform a very crude estimate so as to see the part played by the non-local terms as regards the structure of solitons that might turn around.

The standard procedure to treat the non-linear Schrodinger equation is as follows [9], [10]. We are looking for the solution in the propagating wave (soliton) form 


$$
B=\exp [i(k z-\omega t)] \psi(z-v t)
$$

Introducing (15) into (13) we get two equations for the imaginary and reel parts of the solution (15). From the former one we find the velocity of the soliton $v$

$$
v=2 \omega_{H} k,
$$

and the real part of (13) leads to the equation

$$
\begin{gathered}
\omega \psi=-\omega_{H}\left[-k^{2}+\dot{\psi}\right]-\frac{\lambda}{K} \omega_{T} \psi^{3}-\frac{\lambda}{K} \omega_{T} \psi \int|\psi|^{4} d z^{\prime}+ \\
\frac{\lambda}{K} \omega_{T} \frac{\epsilon a^{2}}{K \Omega^{2}} \psi \int d z^{\prime} \int d z^{\prime \prime} \exp \left(-\mu \mid z^{\prime}-z^{\prime \prime}\right)\left|\psi\left(z^{\prime}\right)\right|^{2}\left|\psi\left(z^{\prime \prime}\right)\right|^{2}+ \\
\frac{\lambda}{K} \omega_{T} \psi \int d z^{\prime} \exp \left(-\mu\left|z-z^{\prime}\right|\right)\left|\psi\left(z^{\prime}\right)\right|^{2}
\end{gathered}
$$

Neglecting $\mu$ we get the standard non-linear Schrodinger equation however with renormalized coefficients. It is easy to find the first integral of the equation, i.e. the energy $W$

$$
(\dot{\psi})^{2}+\left[-k^{2}+\nu-\frac{\lambda}{K} \xi\left(\frac{\epsilon a^{2}}{K \Omega^{2}} C+\Gamma\right)\right] \psi^{2}+\frac{\lambda}{2 K} \xi \psi^{4}=W
$$

Where we used the following notations:

$$
\xi=\frac{\omega_{T}}{\omega_{H}}, \nu=\frac{\omega}{\omega_{H}}, \Gamma=\int d z^{\prime}\left|\psi\left(z^{\prime}\right)\right|^{4}
$$

and $C$ is a factor of the order of 1 . It is worth noting that even though we have made rough simplifications, as regards the non-locality, its bearing on the proton dynamics still has remained, as is seen in the $\epsilon a^{2} / K \Omega^{2}$ term, preserved in the equation given above. One may infer from the fact that under approximations used, there is a profound interplay of the dynamics of proton transfer and the conformational structure of the double helix.

From the equation (17) we obtain the asymptotic width $\Delta$ of the soliton which reads

$$
\Delta=\left[\frac{\omega_{T}^{2}}{\omega_{a c} \omega_{H}} \frac{\epsilon a^{2}}{K \Omega^{2}}\right]^{-1 / 2}
$$


The frequency $\omega_{a c} \equiv K / \hbar$ is generally accepted to be within $10^{11}-10^{13} \mathrm{~Hz}$, but the available estimates for $\omega_{T}, \omega_{H}, \epsilon$ widely diverge, $\omega_{H}=10^{6}-10^{11} \mathrm{~Hz}, \omega_{T} / \omega_{H}=0.1-0.01$, and $\epsilon a^{2} / K \Omega^{2} \simeq 0.1$. Consequently, the width of the solution varies within $10-1000 \AA$, so that one may expect a concerted tunnelling of protons for the lower estimation of $\Delta$.

\section{III.}

Concluding, we should like to draw attention to the fact that the existence of an appreciable interaction between the proton transfer inside the hydrogen bonds of the double helix, and elastic modes of the latter, could result in a concerted dynamics of the protons which is generally of non-linear character and governed by the non-linear and non-local Schrodinger equation. To our knowledge, it is for the first time one is running across the non-linear Schrodinger equation with non-local terms. The transfer of protons may be due to various reasons, and among these are the action of external agents, especially, according to recent experimental work [11], [12] enzymes; it is also worth keeping in mind the possible links with the mutation mechanism, [2]. In this paper we have tried to put these ideas in more quantitative form within the framework of a model that accommodates the basic symmetry structure of the double helix, and as was shown above, allows for certain rough estimates of dynamical features that may surface; presumably of the soliton nature. In this respect, it is worthwhile to note that even the crude estimate we made, conserves the bearing of the double helix, as in seen through the occurrence of the characteristic parameter $\epsilon /\left(K \Omega^{2}\right)$ in the final formulas. Of course the equation (11) we obtained has a larger scope. It may not imply the existence of solitons in DNA, but could open another possibility, the possible existence of nonlinear localization leading to a collapse of an initially broad excitation into a higly localized deformation [13]. Collapse does not occur in the standard NLS equation, but the existence of nonlinearities with higher power in our NLNLS suggest that it could occur in this equation, although, as these terms are non local, no definite conclusion can be given without further studies of the equation. If this hypothesis would be confirmed, a weak and broad perturbation of the hydrogen bonds of a DNA molecule by the vicinity of an enzyme carrying local charges could trigger this nonlinear localization phenomenon and finally lead 
to the formation of a tutomerized form by the tunneling of one proton. As the present stage of the study, this is however only a speculation raised by the form of the NLNLS equation that we derived.

\section{ACKNOWLEDGMENTS}

The research described in this publication was made possible in part by RFFR Grant 00-02-17785. One of the authors (V.L.G.) is thankful to the Lab. Phys. ENS Lyon, for the hospitality, and the programme of exchange between ENS and Landau Institute for Theoretical Physics for the opportunity to participate in the programme. Fruitful discussions with E.Kuznetsov and Yu.M. Yevdokimov, and the useful correspondence with J.L.Leroy and F.Fillaux are gratefully acknowledged. 


\section{REFERENCES}

[1] A.J.Horsewill, N.H.Jones, R.Caciuffo, Science, 291, 100 (2001).

[2] W.Saenger, Principles of Nucleic Acid Structure, Springer - Verlag, New York (1984).

[3] F.H.C. Crick, J. Mol. Biol., 19, 548 (1966).

[4] J.L.Leroy, K.Gehring, A.Kettani, M.Gueron, Biochemistry, 32, 6019 (1993) ; S.Bratos, J.Chem. Phys., 63, 3499 (1975).

[5] N.Bodor, M.J.S.Dewar, A.J.Hagert, J. Am. Chem. Soc., 92, 2929 (1970).

[6] M.Dreyfus, J. Amer. Chem. Soc., 98, 6338 (1976).

[7] V.L.Golo, E.I.Kats, JETP, 84, 1003 (1997); V.L.Golo, Yu.M.Evdokimov, E.I.Kats, JETP Lett., 70, 780 (1999).

[8] T.Dauxois, M.Peyrard, A.R.Bishop, Phys. Rev. E, 47, R44 (1993); T.Dauxois, M.Peyrard, A.R.Bishop, Phys. Rev. E, 47, 684 (1993); T.Dauxois, M.Peyrard, Phys. Rev. E, 51, 1543 (1995).

[9] A.S.Davydov, Sov. Phys. Usp., 251, 898 (1982).

[10] A.C.Scott, Phys.Rev. A, 26, 578 (1982).

[11] D.Antoniou, S.Schwarz, Proc. Nat. Acad. Sci., USA, 94, 12360 (1997).

[12] A.Kohen, P.J.Klinman, Ac. Chem. Res., 31, 397 (1998).

[13] V.E.Zakharov, V.S.Synakh, JETP, 68, 940 (1975); J.J.Rasmuseen, K.Rypdal, Physica Scripta, 33, 481 (1986). 